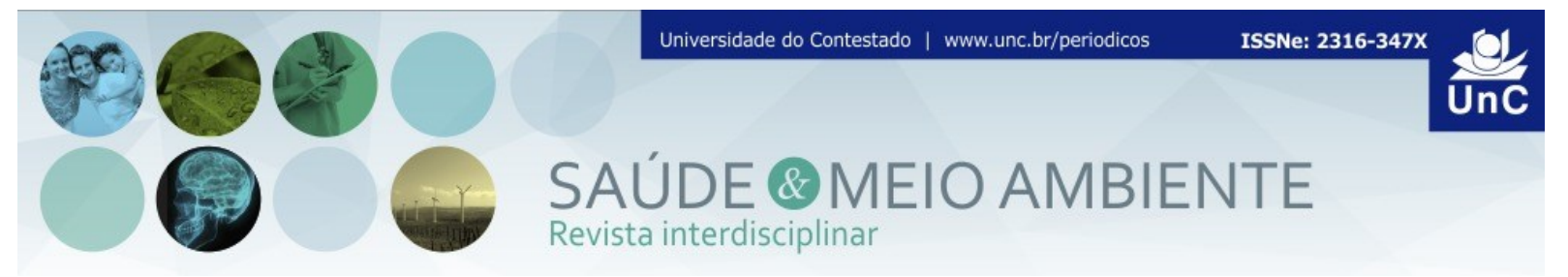

\title{
ASPECTOS SOCIODEMOGRÁFICOS E CAMINHADA COMO FORMA DE DESLOCAMENTO EM IDOSOS
}

\author{
Patricia Broering ${ }^{1}$ \\ Giovane Pereira Balbé2
}

\begin{abstract}
RESUMO
Este estudo teve como objetivo associar aos aspectos sociodemográficos e a caminhada no deslocamento em idosos. Estudo descritivo transversal, realizado com 213 indivíduos residentes na zona urbana da cidade de Rio do Sul - Santa Catarina. Os aspectos sociodemográficos foram avaliados mediante ficha de identificação e a caminhada no deslocamento por meio do Questionário Internacional de Atividade Física, versão longa, aplicados em forma de entrevista. A prevalência de idosos ativos na caminhada como forma de deslocamento foi baixa $(28,2 \%)$. Houve associação significativa na faixa etária $(p=0,021)$, arranjo familiar $(p=0,001)$ e a caminhada. Conclui-se que a maior proporção de idosos fisicamente ativos na caminhada de deslocamento é entre àqueles que moram sozinhos e que essa prevalência tende a reduzir em idosos mais velhos.
\end{abstract}

Palavras-Chave: Atividade física. Envelhecimento. Demografia.

\section{SOCIO-DEMOGRAPHIC ASPECTS AND WALKING AS A MEANS OF LOCOMOTION IN THE ELDERLY}

\begin{abstract}
This study aims to associate walking in locomotion as a way to displacement and demographic aspects. Cross-sectional study conducted with 213 individuals living in the urban area of the city of Rio do Sul - Santa Catarina. Sociodemographic aspects were evaluated through identification form and the walk in the displacement through the International Questionnaire of Physical Activity, long version, applied in the form of an interview. The prevalence of active elderly people in walking as a form of displacement was low (28.2\%). There was a significant association between age $(p=$ $0,021)$, family arrangement $(p=0,001)$ and walking. It is concluded that the largest proportion of physically active elderly people on the walking distance is between those who live alone and that this prevalence tends to reduce in older people.
\end{abstract}

Keywords: Physical activity. Aging. Demography.

\footnotetext{
${ }^{1}$ Graduada em Educação Física. Centro Universitário para o Desenvolvimento do Alto Vale do Itajaí (UNIDAVI). Santa Catarina. Brasil. E-mail: patriciabroering@hotmail.com

${ }_{2}^{2}$ Mestre em Ciências do Movimento Humano. Professor no Centro Universitário para o Desenvolvimento do Alto Vale do Itajaí (UNIDAVI). Santa Catarina. Brasil. E-mail: gbalbe@unidavi.edu.br
} 


\section{INTRODUÇÃO}

A atividade física no deslocamento vem sendo considerada uma estratégia para o aumento dos níveis de comportamento ativo da população em geral (BAUMAN et al., 2012). Evidências na literatura mostram que esse tipo de atividade pode contribuir positivamente à saúde (SANTOS et al., 2009; MADEIRA et al., 2013), especialmente a caminhada, pois é associada ao menor risco de mortalidade por todas as causas (MURTAGH et al., 2015).

Apesar do consenso entre especialistas quanto aos benefícios decorrentes da caminhada no deslocamento, apenas $35 \%$ dos indivíduos acima de 60 anos mantêm-se ativos (SALVADOR; REIS; FLORINDO, 2009; MADEIRA et al., 2013). Dentre os fatores que podem influenciar nesse comportamento, da prática de atividade física, estão as características sociodemográficas (ANTUNES; MAZO; BALBÉ, 2011). Estudo conduzido com 460 sujeitos entre 20 e 79 anos de idade observou uma associação inversa entre atividade física de deslocamento e idade, por outro lado, houve uma relação positiva no deslocamento entre aqueles com estado civil solteiro ou separado, conforme o aumento da idade (PITANGA et al., 2014). Pesquisa realizada com 6506 idosos moradores na zona urbana de 23 estados brasileiros constatou que a proporção de ativos no deslocamento foi menor nas mulheres e idades elevadas. Em contrapartida idosos de cor de pele não branca e com maior escolaridade relataram praticar 150 minutos ou mais de caminhada e uso de bicicleta (MADEIRA et al., 2013). Destaca-se a relevância desta investigação, já que os estudos supracitados apresentam divergências entre os aspectos sociodemográficos associados ao deslocamento e não analisam a caminhada de forma independente. Além disso, a caminhada apresenta baixo impacto, facilidade de acesso e risco reduzido de lesão (SALVADOR; REIS; FLORINDO, 2009), sendo há mais praticada entre idosos, o que reforça a importância de identificar quais aspectos podem contribuir no aumento dessa prática (HANSON; JONES, 2015).

Diante deste contexto, o objetivo deste estudo foi analisar a prevalência de caminhada como forma de deslocamento e associar aos fatores sociodemográficos entre os idosos residentes em Rio do Sul, Santa Catarina (SC).

\section{MÉTODO}

Trata-se de um estudo descritivo e transversal vinculado ao projeto "Atividade Física e Percepção do ambiente em idosos residentes em Rio do Sul", realizado no ano de 2014.

O processo de amostragem foi não probabilístico por acessibilidade. A amostra foi constituída de 213 idosos residentes na zona urbana, conforme lei de zoneamento, da cidade de Rio do Sul, considerados independentes para atividades do cotidiano e com condições físicas e mentais para responder sozinho a entrevista. 
Demais detalhes metodológicos podem ser obtidos no estudo de Balbé, Wathier e Rech (2017).

O estudo foi aprovado pelo Comitê de Ética em Pesquisa (CEP) da Universidade do Vale do Itajaí, conforme parecer $n^{\circ} 685.806$ de 18 de junho de 2014 . Todos os participantes foram informados sobre os objetivos do estudo e tiveram que assinar o Termo de Consentimento Livre e Esclarecido, atendendo aos preceitos éticos vigentes.

Os dados foram coletados por acadêmicos e graduados em Educação Física. A equipe foi treinada pelo coordenador do estudo, para a familiarização com os instrumentos e padronização das condutas de campo. Houve uma testagem dos instrumentos com um grupo de idosos para controle de qualidade dos dados. A coleta foi conduzida na forma de entrevista face a face, realizada no período de julho a outubro de 2014.

Os aspectos sociodemográficos foram avaliados segundo a ficha de identificação, elaborada pelos pesquisadores, a qual identificou as características referentes ao sexo, faixa etária, escolaridade, estado civil, renda familiar, arranjo familiar e ocupação atual.

A caminhada foi avaliada pelo domínio do deslocamento do Questionário Internacional de Atividade Física (IPAQ), versão longa, adaptado e validado para idosos no Brasil (BENEDETTI; MAZO; BARROS, 2004).

A variável referente à caminhada como forma de deslocamento foi tratada em três níveis: inativos (idosos que não atingiram o mínimo de 10 minutos contínuos de atividade física por semana), insuficientemente ativos (que realizaram entre 10 a 149 minutos por semana de atividade física) e ativos (que cumpriram 150 minutos ou mais de atividade física por semana).

Os dados foram adequadamente armazenados em um banco de dados no programa Excel e com um número codificado para cada entrevistado. A análise dos dados ocorreu por meio do pacote estatístico Statistical Package for Social Science (SPSS), versão 17.0. Utilizou-se medidas descritivas (frequência relativa e absoluta) e inferencial mediante o teste qui-quadrado, ou exato de fisher quando necessário, para associação do domínio da atividade física (variável dependente) e dados sociodemográficos (variáveis independentes), conforme o nível de significância de $5 \%$.

\section{RESULTADOS E DISCUSSÃO}

Dos 213 participantes do estudo, a maioria eram mulheres $(78,9 \%)$, entre 60 a 69 anos de idade $(52,1 \%)$, casados $(54,5 \%)$, com até 4 anos de estudos $(56,8 \%)$, renda entre 1 e 2 salários mínimos $(53,1 \%)$, aposentados $(74,6 \%)$ e que residem com o cônjuge $(47,7 \%)$, conforme tabela 1. 
Tabela 1 - Frequência relativa (f) e absoluta (\%) dos aspectos sociodemográficos de idosos residentes em Rio do Sul, SC, Brasil $(n=213)$.

\begin{tabular}{|c|c|c|}
\hline Caraterísticas Sociodemográficas & $f$ & $\%$ \\
\hline \multicolumn{3}{|l|}{ Sexo } \\
\hline Masculino & 45 & 21,1 \\
\hline Feminino & 168 & 78,9 \\
\hline \multicolumn{3}{|l|}{ Faixa Etária (anos) } \\
\hline 60 a 69 & 112 & 52,1 \\
\hline 70 a 79 & 82 & 38,5 \\
\hline 80 ou mais & 19 & 8,9 \\
\hline \multicolumn{3}{|l|}{ Escolaridade (anos de estudo) } \\
\hline 0 a 4 & 121 & 56,8 \\
\hline 5 a 8 & 55 & 25,8 \\
\hline 9 ou mais & 37 & 17,4 \\
\hline \multicolumn{3}{|l|}{ Estado Civil } \\
\hline Solteiro/Viúvo/Separado & 97 & 45,5 \\
\hline Casado & 113 & 54,5 \\
\hline \multicolumn{3}{|l|}{ Renda Atual* (salário mínimo) } \\
\hline$<1$ & 16 & 7,5 \\
\hline 1 a 2 & 113 & 53,1 \\
\hline 3 ou mais & 84 & 39,4 \\
\hline \multicolumn{3}{|l|}{ Arranjo Familiar } \\
\hline Sozinho & 47 & 22,1 \\
\hline Cônjuge ou familiares & 166 & 77,9 \\
\hline \multicolumn{3}{|l|}{ Ocupação Familiar } \\
\hline Aposentado & 159 & 74,6 \\
\hline Aposentado e ativo/Remunerado ativo & 30 & 14,1 \\
\hline Não remunerado & 24 & 11,3 \\
\hline
\end{tabular}

* Salário mínimo em 2014: R\$ 724,00. f= frequência relativa; \%= frequência absoluta.

Quanto a caminhada no deslocamento, observa-se que apenas $28,2 \%(n=60)$ dos idosos foram classificados como ativos (figura 1). 
Figura 1 - Prevalência de caminhada no deslocamento em idosos residentes em Rio do Sul, SC, Brasil $(n=213)$.

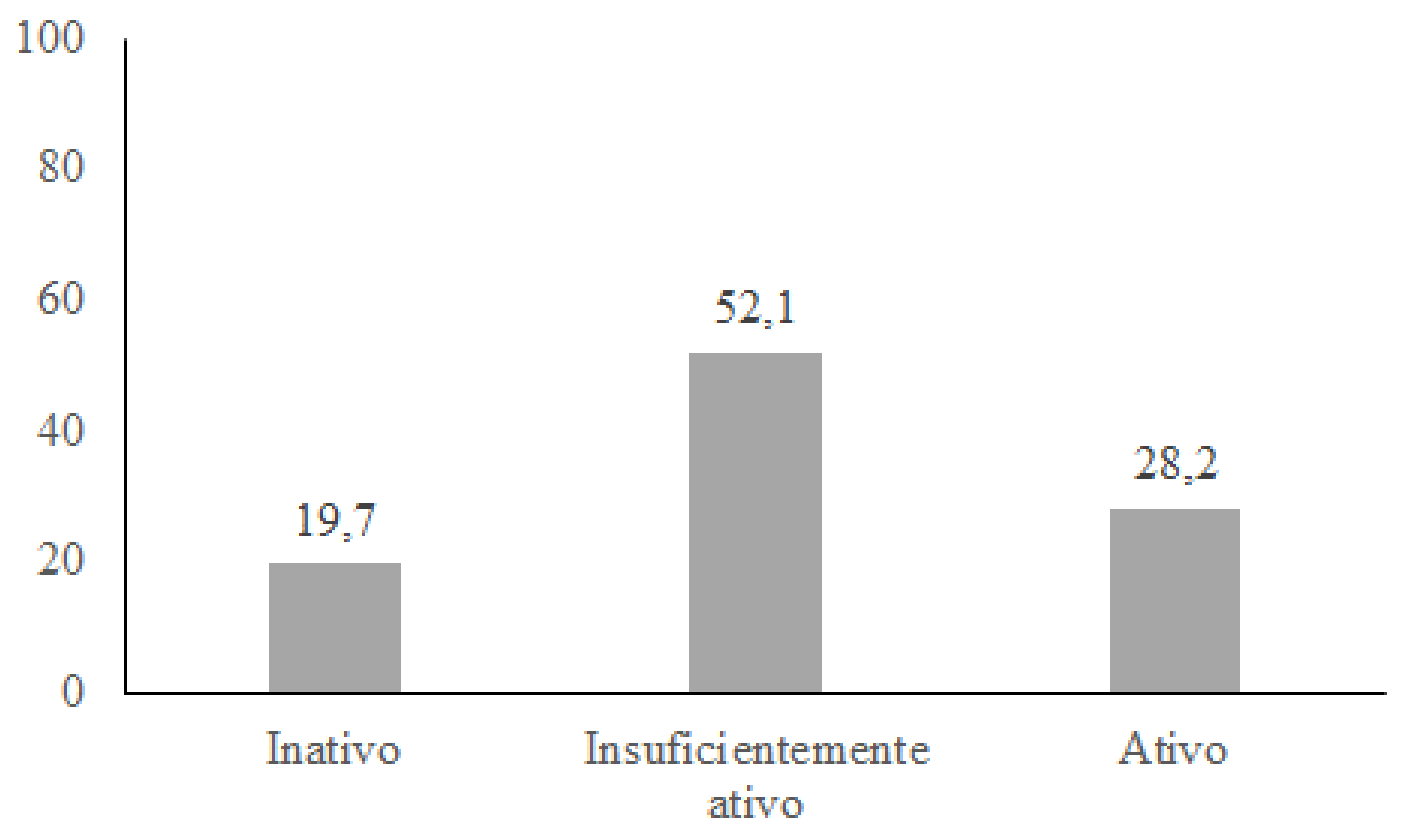

$\mathrm{Na}$ tabela 2, observa-se a associação da caminhada como forma de deslocamento e as características sociodemográficas sendo que $36,8 \%$ dos idosos com 80 anos ou mais apresentaram uma proporção significativamente superior entre os inativos $(p=0,021)$. Em contrapartida, $44,7 \%$ dos idosos que residem sozinhos são ativos $(p=0,001)$. 
Tabela 2 - Associação das características sociodemográficas e caminhada como forma de deslocamento em idosos residentes em Rio do Sul, SC, Brasil $(n=2013)$.

\begin{tabular}{|c|c|c|c|c|}
\hline \multirow{2}{*}{$\begin{array}{l}\text { Características } \\
\text { Sociodemográficas }\end{array}$} & \multicolumn{3}{|c|}{ Caminhada } & \multirow[b]{2}{*}{$p$} \\
\hline & $\begin{array}{c}\text { Inativo } \\
\mathrm{f}(\%)\end{array}$ & $\begin{array}{c}\text { Insuficientemente Ativo } \\
\mathrm{f}(\%)\end{array}$ & $\begin{array}{l}\text { Ativo } \\
\mathrm{f}(\%) \\
\end{array}$ & \\
\hline \multicolumn{5}{|l|}{ Sexo } \\
\hline Masculino & $8(17,8)$ & $22(48,9)$ & $15(33,3)$ & \multirow{2}{*}{0,683} \\
\hline Feminino & $34(20,2)$ & $89(53,0)$ & $45(26,8)$ & \\
\hline \multicolumn{5}{|l|}{ Faixa Etária (anos) } \\
\hline 60 a 69 & $16(14,3)$ & $61(54,5)$ & $35(31,3)$ & \multirow[b]{2}{*}{$0,021^{\alpha}$} \\
\hline $\begin{array}{l}70 \text { a } 79 \\
80 \text { ou mais }\end{array}$ & $\begin{array}{l}19(23,2) \\
7(36,8)\end{array}$ & $\begin{array}{l}41(50,0) \\
9(47,4)\end{array}$ & $\begin{array}{l}22(26,8) \\
3(15,8)\end{array}$ & \\
\hline \multicolumn{5}{|l|}{$\begin{array}{l}\text { Escolaridade (anos de } \\
\text { estudo) }\end{array}$} \\
\hline 0 a 4 & $24(19,8)$ & $68(54,5)$ & $29(24,0)$ & \multirow{3}{*}{0,200} \\
\hline 5 a 8 & $9(16,4)$ & $30(54,5)$ & $16(29,1)$ & \\
\hline 9 ou mais & $9(24,3)$ & $13(35,1)$ & $15(40,5)$ & \\
\hline \multicolumn{5}{|l|}{ Estado Civil } \\
\hline Solteiro/Viúvo/Separado & $19(19,6)$ & $45(46,4)$ & $33(34,0)$ & \multirow{2}{*}{0,193} \\
\hline Casado & $23(19,8)$ & $66(56,9)$ & $27(23,3)$ & \\
\hline \multicolumn{5}{|l|}{$\begin{array}{l}\text { Renda Atual* (salário } \\
\text { mínimo) }\end{array}$} \\
\hline$<1$ & $4(25,0)$ & $12(75,0)$ & $0(0,0)$ & \multirow{3}{*}{$0,485^{\alpha}$} \\
\hline 1 a 2 & $18(15,9)$ & $61(54,0)$ & $34(30,1)$ & \\
\hline 3 ou mais & $20(28,8)$ & $38(45,2)$ & $26(31,0)$ & \\
\hline \multicolumn{5}{|l|}{ Arranjo Familiar } \\
\hline Sozinho & $3(6,4)$ & $23(48,9)$ & $21(44,7)$ & \multirow{2}{*}{$0,001^{\alpha}$} \\
\hline Cônjuge ou Familiar & $39(23,5)$ & $88(53,0)$ & $39(23,5)$ & \\
\hline \multicolumn{5}{|l|}{ Ocupação Atual } \\
\hline Aposentado & $38(23,9)$ & $81(50,9)$ & $40(25,2)$ & \multirow{3}{*}{$0,068^{\alpha}$} \\
\hline Apos. /Ativo/Remunerado & $2(6,7)$ & $13(43,3)$ & $15(50,0)$ & \\
\hline Não remunerado & $2(8,3)$ & $17(70,8)$ & $5(20,8)$ & \\
\hline
\end{tabular}

* Salário mínimo em 2014: R\$ 724,00.

Os dados do estudo apontam predomínio do sexo feminino, de idosos jovens, casados e aposentados. Também de sujeitos que residem com o cônjuge, de baixa renda e grau de escolaridade. A prevalência de caminhada no deslocamento foi de aproximadamente $30 \%$. Associação significativa foi observada com maior proporção de idosos ativos na caminhada de deslocamento entre àqueles que moram sozinhos e que essa prevalência tende a reduzir em idosos mais velhos.

Quanto as características da amostra, de forma geral, os resultados são semelhantes ao observado em outros estudos (MAZO et al., 2005; STREIT et al., 2015). Há supremacia de mulheres idosas, neste estudo, é um fenômeno conhecido como "feminização da velhice", que acompanha o envelhecimento populacional no mundo, sendo mais evidente em faixas etárias superiores (NERI, 2007). Já o maior percentual de idosos jovens, casados, aposentados e de baixa renda e escolaridade 
reforçam o padrão sociodemográfico nos estudos com essa população, inclusive com idosos de Santa Catarina (MAZO et al., 2005).

Em relação a caminhada, constatou-se em nosso estudo baixa prevalência de idosos ativos no deslocamento, fato também observado em outras pesquisas (MADEIRA et al., 2013; SALVADOR; REIS; FLORINDO, 2009). Por outro lado, a Vigilância de fatores de risco e proteção para doenças crônicas por inquérito telefônico (VIGITEL) no conjunto das 27 capitais brasileiras, identificou $5 \%$ de prevalência de caminhada ou uso de bicicleta como forma de deslocamento na faixa etária acima dos 65 anos ou mais (BRASIL, 2017). Acredita-se que essa diferença esteja atrelada as características metodológicas dos estudos, bem como, por uma possível influência do ambiente, embora não avaliado, já que cidades de pequeno porte, caso deste estudo, não favoreça o deslocamento ativo em função da proximidade dos locais (MADEIRA et al., 2013).

Entre os aspectos associados destaca-se o arranjo familiar, sendo que a maior proporção de idosos fisicamente ativos é entre àqueles que moram sozinhos. Acredita-se que o fato de o idoso não ter um companheiro(a) exige de sua independência e autonomia para realização da caminhada como meio de deslocamento, já que quase três milhões de idosos moram sozinhos no Brasil (IBGE, 2007) e como consequência parece contribuir para atividade física de deslocamento. Tal hipótese é reforçada por Mazo et al. (2005) que destacam a caminhada como meio de transporte para realizar atividades cotidianas como às compras, à visita a parentes e vizinhos e à ida ao Grupo de Convivência para Idosos.

Com relação à idade, nota-se que quanto maior a faixa etária do idoso menos ativo fisicamente ele se torna. Isso pode ser explicado devido a perda da capacidade funcional advinda com o avanço da idade (GIEHL et al., 2012), pois a redução na mobilidade tende a limitar a condição de caminhada do idoso já que o mesmo tende a utilizar outras formas de deslocamento para se deslocar de um local a outro, como carro ou ônibus (MAZO et al., 2005; QUEIROZ, et al., 2014).

Os níveis de inatividade física podem aumentar conforme o avanço da idade, já que idosos mais velhos tendem a apresentar maior número de doenças decorrente do processo de envelhecimento (STREIT et al., 2015). Outros fatores também associados a redução nos níveis de atividade física entre idosos são a perda de massa muscular e presença de artrose e/ou artrite, fatores estes, que dificultam ou impedem a realização de movimento, contribuindo para inatividade em idosos longevos (QUEIROZ, et al., 2014).

Franchi e Montenegro (2005) relatam que há sim diminuição no nível de atividade física com o envelhecimento, mas afirmam que, por outro lado, os praticantes tendem a realizar a caminhada de forma mais prevalente. A caminhada é a principal atividade física que pode ser incorporada ao cotidiano dos idosos por ser potencialmente mais acessível e indicada em programas de promoção de saúde para esta população (SALVADOR; REIS; FLORINDO, 2009). Além disso, é uma atividade de fácil acesso, baixo custo e reduzido risco de lesão (HANSON; JONES, 2015). 
No Brasil, os estudos relacionados com a prática de atividade física em idosos no âmbito do deslocamento ainda são escassos (SALVADOR; REIS; FLORINDO, 2009). A maior parte dos levantamentos realizados apresentam falta de padronização metodológica quanto ao uso de instrumentos, pontos de corte e seleção amostral (SANTOS, et al., 2009). O presente estudo apresenta algumas limitações como a medida indireta de caminhada no deslocamento e o fato de não considerar as características ambientais que podem influenciar no deslocamento dessa população. Entretanto, houve dificuldade na comparação entre outros estudos com essa temática, já que a atividade física de deslocamento pesquisada não ocorre de forma isolada.

\section{CONSIDERAÇÕES FINAIS}

Os resultados obtidos pela presente pesquisa apontaram baixa prevalência de atividade física entre os idosos residentes na cidade de Rio do Sul. Constatou-se também, que a maioria dos participantes do estudo eram mulheres, entre 60 a 69 anos de idade, casados, com até 4 anos de estudos, renda entre 1 e 2 salários mínimos, aposentados e que residem com o cônjuge.

Conclui-se desta forma, que a maior proporção de idosos fisicamente ativos na caminhada de deslocamento é entre àqueles que moram sozinhos e que essa prevalência tende a reduzir em idosos mais velhos. Presume-se que o fato de o idoso residir sozinho induza a maior independência e autonomia na realização de suas tarefas diárias. Instiga-se desta forma, a realização de novos estudos nessa área considerando o arranjo familiar e a idade, com vista a auxiliar a construção de políticas públicas eficazes no aumento do nível de atividade física no deslocamento em idosos.

\section{REFERÊNCIAS}

Antunes G, Mazo G Z, Balbé G P. Relação da autoestima entre a percepção de saúde e aspectos sociodemográficos de idosos praticantes de exercício físico. Revista da Educação Física/UEM. 2011; 22(4): 583-589.

Balbé G P, Wathier C A, Rech C R. Características do ambiente do bairro e prática de caminhada no lazer e deslocamento em idosos. Revista Brasileira de Atividade Física \& Saúde, Pelotas. 2017; 22(2): 195-205.

Bauman A E, Reis R S, Sallis J F, Wells J C, Loos R J, Martin B W et al. Correlates of physical activity: why are some people physically active and others not? Lancet. 2012; 380(9838):258-71. 
Benedetti T R B, Mazo G Z, Barros M V G. Aplicação do questionário internacional de Atividade física em mulheres idosas: validade concorrente e reprodutibilidade teste e reteste. Revista Brasileira de Ciência e Movimento. Brasília. 2004; 12(1): 2534.

Brasil. Vigitel Brasil 2016: Vigilância de fatores de risco e proteção para doenças crônicas por inquérito telefônico: estimativas sobre frequência e distribuição sociodemográfica de fatores de risco e proteção para doenças crônicas nas capitais dos 26 estados brasileiros e no Distrito Federal em 2016. Ministério da Saúde, Secretaria de Vigilância em Saúde, Departamento de Vigilância de Doenças e Agravos não Transmissíveis e Promoção da Saúde. Brasília: Ministério da Saúde; 2017.

Franchi K M B, Montenegro R M M. Atividade Física: uma necessidade para a boa saúde na terceira idade. Revista Brasileira em Promoção da Saúde. Fortaleza. 2005; 18(3); 152-156.

Giehl M, Schneider I J C, Corseuil HX, Benedetti T R B, D’orsi E. Atividade física e percepção do ambiente em idosos: estudo populacional em Florianópolis. Revista de Saúde Pública. São Paulo. 2012; 46(3): 516-525.

Hanson, S.; Jones, A. Is there evidence that walking groups have health benefits? A systematic review and meta-analysis. Br J Sports Med. 2015; 0:1-7.

IBGE (Instituto Brasileiro de Geografia e Estatística). Síntese de Indicadores Sociais. 2007. [cited 2018 jun 02]. Available from:

http://biblioteca.ibge.gov.br/visualizacao/livros/liv36151.pdf

Madeira M C, Siqueira F C V, Facchini L A, Silveira D S, Tomasi E, Thumé E et al. Atividade física no deslocamento em adultos e idosos do Brasil: prevalências e fatores associados. Cadernos da Saúde Pública. Rio de Janeiro. 2013; 29(1): 165174.

Mazo G Z, Mota J, Gonçalves L H T, Matos M G. Nível de atividade física, condições de saúde e características sociodemográficas de mulheres idosas brasileiras.

Revista Port. Desp. 2005; 5: 202-212.

Murtagh E M, Nichols L, Mohammed M A, Holder R, Nevill A M, Murphy M H. The effect of walking on risk factors for cardiovascular disease: an updated systematic review and meta-analysis of randomised control trials. Prev Med. 2015; 72:34-43, 2015.

Pitanga F G, Beck C C, Pitanga C P S, Freitas M M, Almeida L A B. Prevalência e fatores sociodemográficos e ambientais associados à atividade física no tempo livre e no deslocamento em adultos. Motricidade. Ribeira de Pena, 2014; 10(1): 3-13.

Queiroz B M, Coqueiro R S, Neto J S L, Borgatto A F, Barbosa A R, Fernandes M H. Inatividade Física em idosos não institucionalizados: estudo de base populacional. Revista Ciência e Saúde Coletiva. 2014; 19(08): 3489-3496. 
Salvador E P, Reis R S, Florindo A A. A prática de caminhada como forma de deslocamento e sua associação com a percepção do ambiente em idosos. Revista Brasileira de Atividade Física \& Saúde. 2009; 14(3): 197-205.

Santos C M, Barbosa J M V, Cheng L A, Júnior R S W, Barros M V G. Atividade física no contexto dos deslocamentos: revisão sistemática dos estudos epidemiológicos realizados no Brasil. Revista Brasileira de Atividade Física \& Saúde. 2009; 14(1):15-22.

Streit I A, Benetti M Z, Mota J A OS, Mazo G Z Nível de atividade física e condições de saúde em idosos centenários. Do corpo: ciências e artes. 2015; 5(1):1-10.

Artigo recebido em: 23/03/2018

Artigo aprovado em: 05/10/2018

Artigo publicado em: 27/03/2019 